Supporting Information

\title{
Smart Functionalization of Ceramic-Coated AZ31
}

\section{Magnesium Alloy}

Beatriz Mingo*, Yue Guo, Rafael Leiva-Garcia, Brian Connolly, Allan Matthews and Aleksey Yerokhin

Department of Materials, The University of Manchester, Oxford Road, Manchester, M13 9PL, UK.

* beatriz.mingo@manchester.ac.uk 
1. SEM characterization of PEO coatings

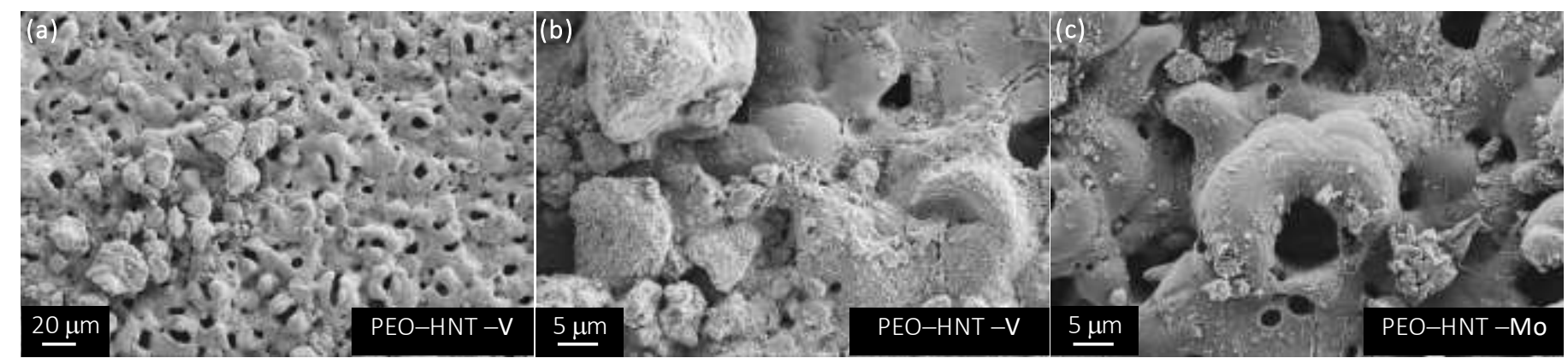

Figure S1. SEM micrographs of (a, b) PEO-HNT-V and (c) PEO-HNT-Mo coatings 
2. Electrochemical Impedance Spectroscopy (EIS) study
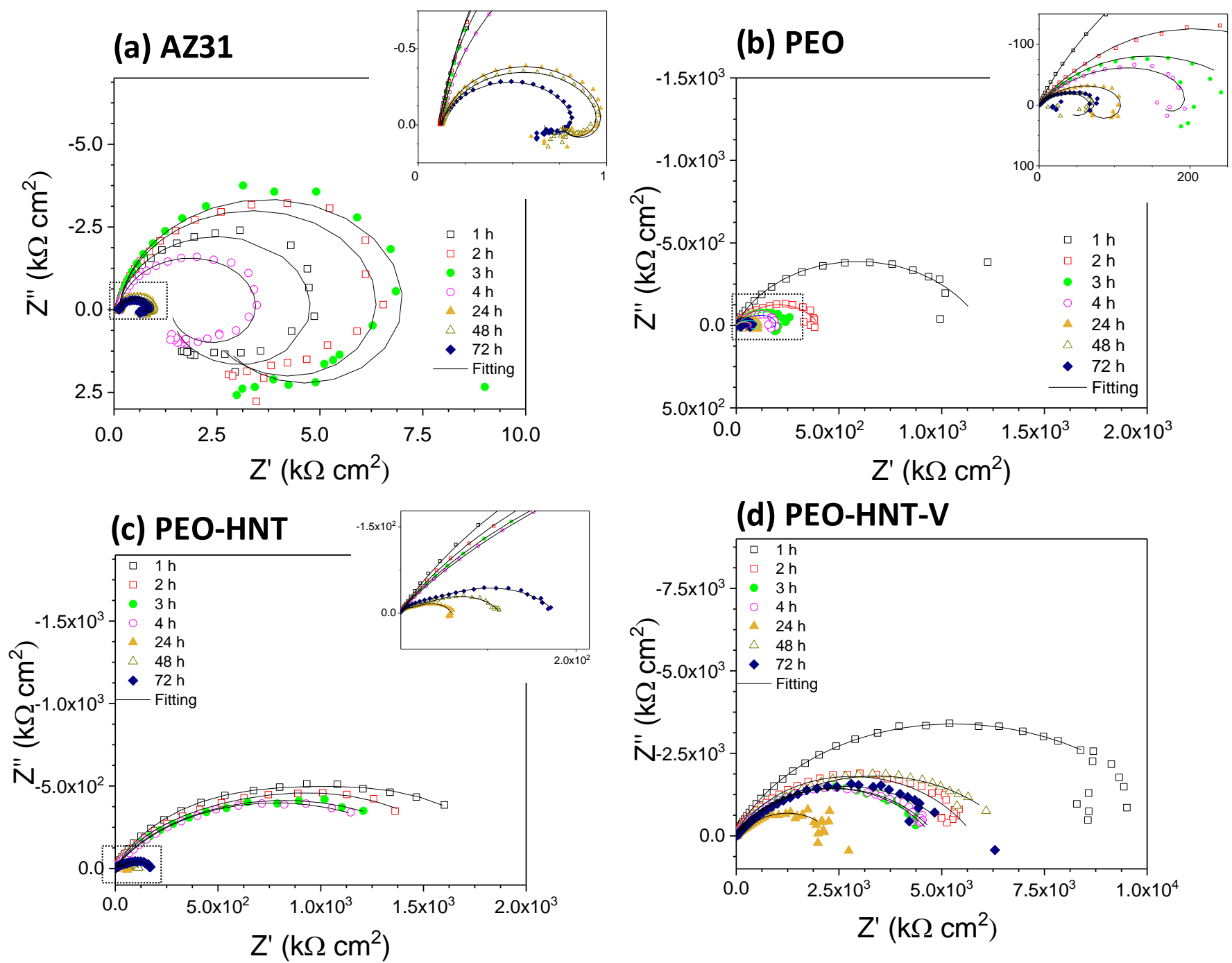

\section{(d) PEO-HNT-V}

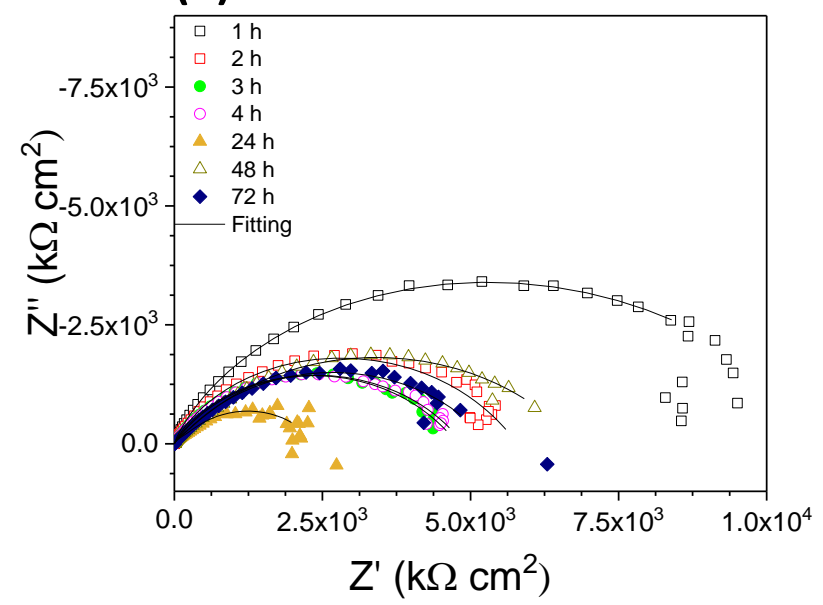

\section{(e) PEO-HNT-Mo}

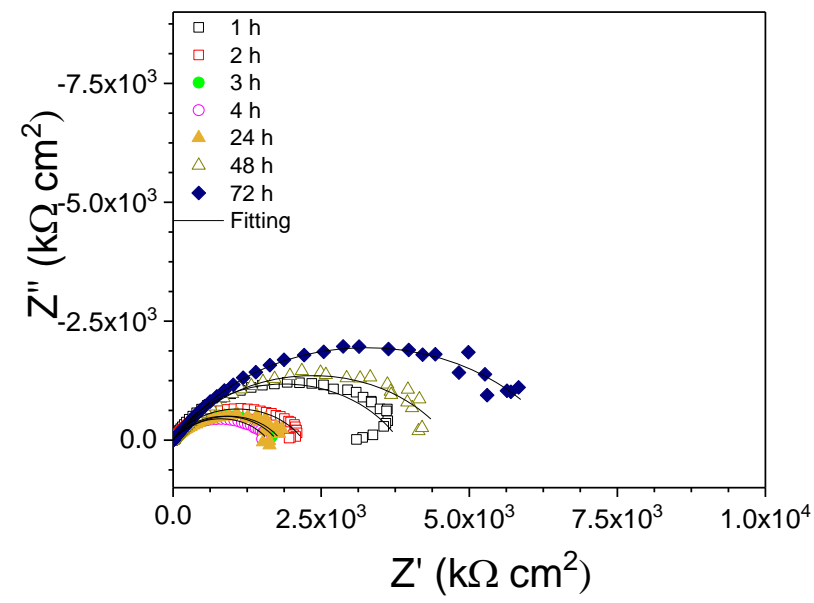

(f) PEO-HNT-HQ

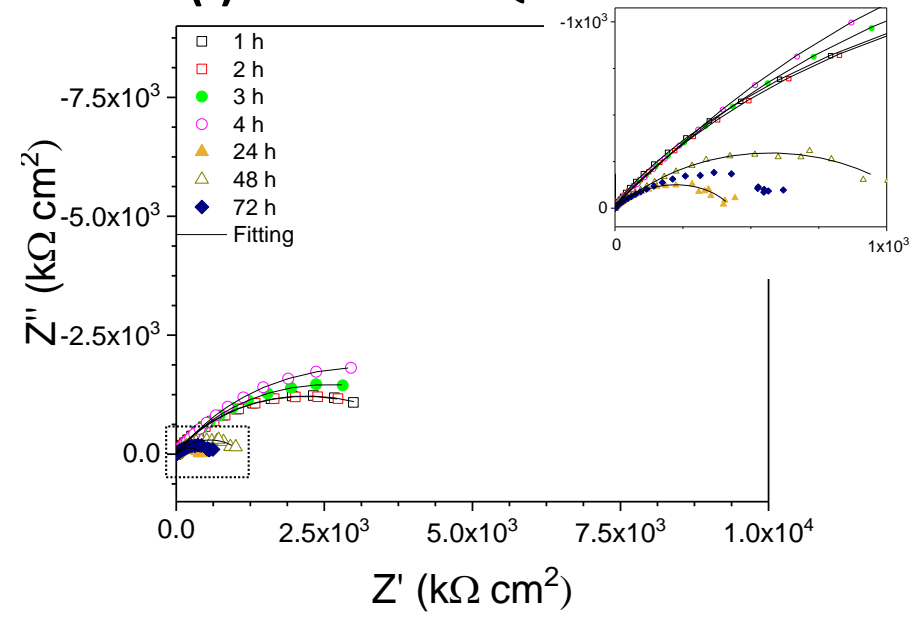

Figure S2. Nyquist diagrams of the studied coatings after $1-72 \mathrm{~h}$ of immersion in naturally aerated $0.5 \mathrm{wt}$. \% $\mathrm{NaCl}$ 
Table S1. EC fitting results for impedance spectra of AZ31 alloy immersed for up to $72 \mathrm{~h}$ in $0.5 \% \mathrm{NaCl}$ solutions

\begin{tabular}{|c|c|c|c|c|c|}
\hline Time (h) & $\mathbf{C P E}_{\mathbf{d l}-\mathbf{T}}\left(\mathbf{S ~ s}^{\mathbf{n}} \mathbf{~ c m}^{2}\right)$ & $\mathbf{C P E}-\mathbf{n}$ & $\mathbf{R}_{\mathbf{c t}}\left(\mathbf{k} \boldsymbol{\Omega} \mathbf{~ c m}^{\mathbf{2}}\right)$ & $\left.\mathbf{L} \mathbf{( k \boldsymbol { \Omega } \mathbf { ~ s m }} \mathbf{~}^{\mathbf{2}}\right)$ & $\mathbf{R}_{\mathbf{L}}\left(\mathbf{k} \boldsymbol{\Omega} \mathbf{~ c m}^{\mathbf{2}}\right)$ \\
\hline $1 \mathrm{~h}$ & $4.59 \times 10^{-6}$ & 0.931 & 5.09 & 1.57 & 2.48 \\
\hline $2 \mathrm{~h}$ & $4.82 \times 10^{-6}$ & 0.932 & 5.89 & 1.75 & 2.39 \\
\hline $3 \mathrm{~h}$ & $6.17 \times 10^{-6}$ & 0.930 & 4.71 & 1.80 & 2.82 \\
\hline $4 \mathrm{~h}$ & $7.04 \times 10^{-6}$ & 0.926 & 3.90 & 1.41 & 2.72 \\
\hline $24 \mathrm{~h}$ & $1.19 \times 10^{-5}$ & 0.921 & 2.49 & 3.13 & 4.58 \\
\hline $48 \mathrm{~h}$ & $1.47 \times 10^{-5}$ & 0.859 & 1.55 & 1.26 & 3.88 \\
\hline $72 \mathrm{~h}$ & $1.64 \times 10^{-5}$ & 0.822 & 1.30 & 0.84 & 3.36 \\
\hline
\end{tabular}

Table S2. EC fitting results for impedance spectra of PEO coatings immersed for up to $72 \mathrm{~h}$ in $0.5 \% \mathrm{NaCl}$ solutions

\begin{tabular}{|c|c|c|c|c|c|c|c|c|}
\hline Time (h) & $\mathbf{C P E}_{\text {in- }} \mathbf{T}\left(\mathbf{S ~ s}^{\mathbf{n}} \mathbf{c m}^{2}\right)$ & $\mathbf{C P E}-\mathbf{n}$ & $\mathbf{R}_{\text {in }}\left(\mathbf{k} \boldsymbol{\Omega} \mathbf{c m}^{2}\right)$ & $\mathbf{C P E}_{\mathrm{dl}}-\mathbf{T}\left(\mathbf{S ~ s}^{\mathbf{n}} \mathbf{c m}^{2}\right)$ & $\begin{array}{c}\mathbf{C P E}- \\
\mathbf{n}\end{array}$ & $\begin{array}{c}\mathbf{R}_{\mathbf{c t}}(\mathbf{k} \boldsymbol{\Omega} \\
\left.\mathbf{c m}^{2}\right)\end{array}$ & $\begin{array}{c}\mathbf{L}(\mathbf{k} \boldsymbol{\Omega} \\
\left.\mathbf{s} \mathbf{~ c m}^{2}\right)\end{array}$ & $\begin{array}{c}\mathbf{R}_{\mathbf{L}}(\mathbf{k} \boldsymbol{\Omega} \\
\left.\mathbf{c m}^{2}\right)\end{array}$ \\
\hline $1 \mathrm{~h}$ & $1.61 \times 10^{-7}$ & 0.777 & 894.92 & $6.43 \times 10^{-7}$ & 0.710 & 274.81 & & \\
\hline $2 \mathrm{~h}$ & $3.11 \times 10^{-7}$ & 0.673 & 379.40 & $2.03 \times 10^{-7}$ & 0.998 & 66.08 & & \\
\hline $3 \mathrm{~h}$ & $1.41 \times 10^{-7}$ & 0.744 & 17.56 & $4.04 \times 10^{-7}$ & 0.571 & 293.11 & & \\
\hline $4 \mathrm{~h}$ & $3.19 \times 10^{-8}$ & 0.874 & 5.18 & $6.99 \times 10^{-7}$ & 0.563 & 247.96 & 487.54 & 306.41 \\
\hline $24 \mathrm{~h}$ & $1.43 \times 10^{-8}$ & 0.929 & 1.89 & $8.60 \times 10^{-7}$ & 0.561 & 128.95 & 127.32 & 121.96 \\
\hline $48 \mathrm{~h}$ & $2.49 \times 10^{-7}$ & 0.759 & 6.42 & $1.80 \times 10^{-7}$ & 0.916 & 39.89 & 90.50 & 49.31 \\
\hline $72 \mathrm{~h}$ & $1.40 \times 10^{-7}$ & 0.887 & 0.13 & $9.56 \times 10^{-7}$ & 0.890 & 33.96 & 87.33 & 29.72 \\
\hline
\end{tabular}

Table S3. EC fitting results for impedance spectra of PEO-HNT coatings immersed for up to $72 \mathrm{~h}$ in $0.5 \%$ $\mathrm{NaCl}$ solutions

\begin{tabular}{|c|c|c|c|c|c|c|}
\hline Time $(\mathbf{h})$ & $\mathbf{C P E}_{\text {in }} \mathbf{T}\left(\mathbf{S ~ s}^{\mathbf{n}} \mathbf{~ c m}^{2}\right)$ & $\mathbf{C P E}-\mathbf{n}$ & $\mathbf{R}_{\text {in }}\left(\mathbf{k} \boldsymbol{\Omega} \mathbf{~ c m}^{2}\right)$ & $\mathbf{C P E}$ dl- $\mathbf{T}\left(\mathbf{S ~ s}^{\mathbf{n}} \mathbf{c m}^{\mathbf{2}}\right)$ & $\mathbf{C P E}-\mathbf{n}$ & $\mathbf{R}_{\mathbf{c t}}\left(\mathbf{k} \boldsymbol{\Omega} \mathbf{~ c m}^{2}\right)$ \\
\hline $1 \mathrm{~h}$ & $1.33 \times 10^{-7}$ & 0.737 & 1343.60 & $1.22 \times 10^{-6}$ & 0.801 & 591.03 \\
\hline $2 \mathrm{~h}$ & $1.88 \times 10^{-7}$ & 0.706 & 1104.60 & $8.98 \times 10^{-7}$ & 0.713 & 634.09 \\
\hline $3 \mathrm{~h}$ & $2.21 \times 10^{-7}$ & 0.691 & 762.12 & $3.78 \times 10^{-7}$ & 0.662 & 699.77 \\
\hline $4 \mathrm{~h}$ & $2.46 \times 10^{-7}$ & 0.682 & 460.04 & $3.17 \times 10^{-7}$ & 0.470 & 1224.00 \\
\hline $24 \mathrm{~h}$ & $1.71 \times 10^{-7}$ & 0.714 & 26.77 & $7.45 \times 10^{-7}$ & 0.746 & 36.20 \\
\hline $48 \mathrm{~h}$ & $1.78 \times 10^{-7}$ & 0.706 & 46.23 & $8.55 \times 10^{-7}$ & 0.749 & 67.81 \\
\hline $72 \mathrm{~h}$ & $1.35 \times 10^{-7}$ & 0.725 & 59.25 & $8.42 \times 10^{-7}$ & 0.679 & 117.44 \\
\hline
\end{tabular}


Table S4. EC fitting results for impedance spectra of PEO-HNT-V coatings immersed for up to $72 \mathrm{~h}$ in $0.5 \% \mathrm{NaCl}$ solutions

\begin{tabular}{|c|c|c|c|c|c|c|}
\hline Time (h) & $C P E_{i n}-T\left(S s^{n} \mathbf{c m}^{2}\right)$ & CPE-n & $R_{\text {in }}\left(k \Omega \mathbf{c m}^{2}\right)$ & $C P E_{d l}-T\left(S s^{n} \mathbf{c m}^{2}\right)$ & CPE-n & $\mathbf{R}_{\mathrm{ct}}\left(\mathrm{k} \Omega \mathbf{c m}^{2}\right)$ \\
\hline $1 \mathrm{~h}$ & $1.93 \times 10^{-8}$ & 0.883 & 383.53 & $3.35 \times 10^{-8}$ & 0.608 & 9756.80 \\
\hline $2 \mathrm{~h}$ & $2.39 \times 10^{-8}$ & 0.866 & 203.14 & $5.36 \times 10^{-8}$ & 0.615 & 5593.00 \\
\hline $3 \mathrm{~h}$ & $2.74 \times 10^{-8}$ & 0.853 & 171.73 & $7.54 \times 10^{-8}$ & 0.616 & 4607.40 \\
\hline $4 \mathrm{~h}$ & $3.19 \times 10^{-8}$ & 0.837 & 195.93 & $9.04 \times 10^{-8}$ & 0.619 & 4683.10 \\
\hline $24 \mathrm{~h}$ & $4.65 \times 10^{-8}$ & 0.800 & 361.30 & $1.57 \times 10^{-7}$ & 0.719 & 1871.90 \\
\hline $48 \mathrm{~h}$ & $5.02 \times 10^{-8}$ & 0.789 & 392.16 & $1.54 \times 10^{-7}$ & 0.590 & 6407.70 \\
\hline $72 \mathrm{~h}$ & $5.81 \times 10^{-8}$ & 0.776 & 306.82 & $1.47 \times 10^{-7}$ & 0.605 & 5389.60 \\
\hline
\end{tabular}

Table S5. EC fitting results for impedance spectra of PEO-HNT-Mo coatings immersed for up to $72 \mathrm{~h}$ in $0.5 \% \mathrm{NaCl}$ solutions

\begin{tabular}{|c|c|c|c|c|c|c|}
\hline Time (h) & $\mathrm{CPE}_{\mathrm{in}}-\mathrm{T}\left(\mathrm{S} \mathrm{s}^{\mathrm{n}} \mathrm{cm}^{2}\right)$ & CPE-n & $R_{\text {in }}\left(\mathbf{k} \Omega \mathbf{c m}^{2}\right)$ & $\mathrm{CPE}_{\mathrm{dl}}-\mathrm{T}\left(\mathrm{S} \mathrm{s}^{\mathrm{n}} \mathbf{c m}^{2}\right)$ & CPE-n & $\mathbf{R}_{\mathrm{ct}}\left(\mathbf{k} \Omega \mathbf{c m}^{2}\right)$ \\
\hline $1 \mathrm{~h}$ & $2.37 \times 10^{-8}$ & 0.873 & 105.21 & $7.90 \times 10^{-8}$ & 0.641 & 3711.00 \\
\hline $2 \mathrm{~h}$ & $3.06 \times 10^{-8}$ & 0.853 & 89.71 & $1.23 \times 10^{-7}$ & 0.634 & 2110.10 \\
\hline $3 \mathrm{~h}$ & $3.67 \times 10^{-8}$ & 0.837 & 91.09 & $1.68 \times 10^{-7}$ & 0.622 & 1660.70 \\
\hline $4 \mathrm{~h}$ & $4.36 \times 10^{-8}$ & 0.820 & 103.31 & $2.01 \times 10^{-7}$ & 0.615 & 1502.30 \\
\hline $24 \mathrm{~h}$ & $7.14 \times 10^{-8}$ & 0.768 & 341.69 & $2.10 \times 10^{-7}$ & 0.628 & 1476.00 \\
\hline $48 \mathrm{~h}$ & $7.74 \times 10^{-8}$ & 0.755 & 411.99 & $1.18 \times 10^{-7}$ & 0.628 & 4253.50 \\
\hline $72 \mathrm{~h}$ & $7.57 \times 10^{-8}$ & 0.762 & 253.02 & $1.21 \times 10^{-7}$ & 0.648 & 6252.80 \\
\hline
\end{tabular}

Table S6. EC fitting results for impedance spectra of PEO-HNT-8-HQ coatings immersed for up to $72 \mathrm{~h}$ in $0.5 \% \mathrm{NaCl}$ solutions

\begin{tabular}{|c|c|c|c|c|c|c|}
\hline Time $(\mathbf{h})$ & $\mathbf{C P E}_{\mathbf{i n}}-\mathbf{T}\left(\mathbf{S ~ s}^{\mathbf{n}} \mathbf{c m}^{2}\right)$ & $\mathbf{C P E}-\mathbf{n}$ & $\mathbf{R}_{\text {in }}\left(\mathbf{k} \boldsymbol{\Omega} \mathbf{~ c m}^{\mathbf{2}}\right)$ & $\mathbf{C P E}$ dl-T $\left(\mathbf{S ~ s}^{\mathbf{n}} \mathbf{c m}^{\mathbf{2}}\right)$ & $\mathbf{C P E}-\mathbf{n}$ & $\mathbf{R}_{\mathbf{c t}}\left(\mathbf{k} \boldsymbol{\Omega} \mathbf{c m}^{2}\right)$ \\
\hline $1 \mathrm{~h}$ & $2.11 \times 10^{-8}$ & 0.881 & 198.79 & $1.15 \times 10^{-7}$ & 0.608 & 6688.90 \\
\hline $2 \mathrm{~h}$ & $2.61 \times 10^{-8}$ & 0.864 & 174.58 & $1.42 \times 10^{-7}$ & 0.608 & 7463.70 \\
\hline $3 \mathrm{~h}$ & $3.08 \times 10^{-8}$ & 0.849 & 174.38 & $1.57 \times 10^{-7}$ & 0.608 & 8630.70 \\
\hline $4 \mathrm{~h}$ & $3.33 \times 10^{-8}$ & 0.843 & 170.66 & $1.67 \times 10^{-7}$ & 0.609 & 9725.20 \\
\hline $24 \mathrm{~h}$ & $1.62 \times 10^{-7}$ & 0.728 & 85.811 & $2.63 \times 10^{-7}$ & 0.678 & 343.75 \\
\hline $48 \mathrm{~h}$ & $1.58 \times 10^{-7}$ & 0.730 & 47.249 & $2.13 \times 10^{-7}$ & 0.835 & 76.99 \\
\hline $72 \mathrm{~h}$ & $1.64 \times 10^{-7}$ & 0.719 & 31.026 & $2.88 \times 10^{-7}$ & 0.904 & 27.15 \\
\hline
\end{tabular}

